OPEN ACCESS

Edited by:

Hung-Ming Chang,

Taipei Medical University, Taiwan

Reviewed by:

Wenchieh Liao,

Chung Shan Medical University,

Taiwan

Tzer-Bin Lin,

Taipei Medical University, Taiwan

*Correspondence:

Jing Wu

boaz.jing@gmail.com

tThese authors have contributed equally to this work

Received: 30 August 2018 Accepted: 30 October 2018 Published: 19 November 2018

Citation:

Fan Y, Du L, Fu Q, Zhou Z, Zhang J, Li G and Wu J (2018) Inhibiting the NLRP3 Inflammasome

With MCC950 Ameliorates

Isoflurane-Induced Pyroptosis and Cognitive Impairment in Aged Mice. Front. Cell. Neurosci. 12:426. doi: 10.3389/fncel.2018.00426

\section{Inhibiting the NLRP3 Inflammasome With MCC950 Ameliorates Isoflurane-Induced Pyroptosis and Cognitive Impairment in Aged Mice}

\author{
Yunxia Fan't, Liwu Durt, Qun Fu', Zhiqiang Zhou's, Jingyu Zhang', Guomin $\mathrm{Li}^{1}$ and \\ Jing $W u^{4 *}$
}

${ }^{1}$ Department of Anesthesiology, Jintan Hospital, Jiangsu University, Changzhou, China, ${ }^{2}$ Department of Anesthesiology, Nanjing Branch of Shanghai Changzheng Hospital, The Second Military Medical University, Nanjing, China, ${ }^{3}$ Department of Anesthesiology, Jinling Clinical Medical College of Nanjing Medical University, Nanjing, China, ${ }^{4}$ Jiangsu Key Laboratory of Molecular Medicine, Medical School of Nanjing University, Nanjing, China

Nod-like receptor protein 3 (NLRP3) inflammasome activation has been implicated in the pathogenesis of general anesthesia (GA)-induced neuroinflammation and cognitive impairment in aged rodents. However, the cellular basis for cognitive impairment is still not fully understood, and effective pharmacologic agents targeting the NLRP3 inflammasome during GA are lacking. This study explores the protective effects of the NLRP3 inflammasome inhibitor MCC950 on pyroptosis and cognitive impairment in aged mice exposed to isoflurane. Seventy-two 15-month-old male C57BL/6 mice were randomized to receive $2 \mathrm{~h}$ of $1.5 \%$ isoflurane plus $30 \%$ oxygen $\left(\mathrm{O}_{2}\right)$ or $30 \% \mathrm{O}_{2}$ alone, respectively. MCC950 (10 mg/kg) or vehicle was intraperitoneally administered 30 min before gas inhalation. Brain tissues were harvested for histochemical analysis and biochemical assays. Learning and memory abilities were evaluated by behavioral tests. We found that isoflurane GA caused upregulations of hippocampal NLRP3,

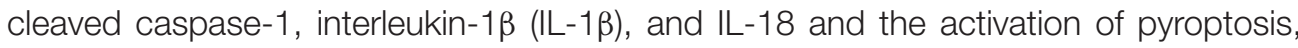
which is NLRP3 inflammasome-dependent; this consequently gave rise to neuronal damage and cognitive impairment in aged mice. Interestingly, pretreatment with NLRP3 inflammasome inhibitor MCC950 not only provided a neuroprotective effect against the inflammasome activation but also ameliorated pyroptosis and cognitive impairment in aged mice exposed to isoflurane. Our data demonstrate that pyroptosis is involved in NLRP3 inflammasome-mediated isoflurane-induced cognitive impairment in aged mice and suggest that inhibiting the NLRP3 inflammasome with MCC950 may have clinically therapeutic benefits for elderly patients undertaking GA.

Keywords: NLRP3, neuroinflammation, pyroptosis, cognitive impairment, general anesthesia, aging

\section{INTRODUCTION}

Postoperative cognitive dysfunction (POCD) is a common complication associated with significant morbidity and mortality in elderly patients (Berger et al., 2015; Moskowitz et al., 2017). General anesthesia (GA)-induced neuroinflammation is increasingly emphasized as a key factor in the pathogenesis of POCD (Hovens et al., 2014; Umholtz and Nader, 2017; Skvarc et al., 2018). 
The NOD-like receptor protein 3 (NLRP3) inflammasome, an intracellular multiprotein complex, is the best recognized and most widely studied inflammasome. It regulates the cleavage of caspase-1, which controls the processing and secretion of the pro-inflammatory cytokines IL-1 $\beta$ and IL-18 (He et al., 2016a; Dempsey et al., 2017; Jiang et al., 2018). These cytokines are crucial in initiating or amplifying the innate immune response and neuroinflammation following GA. We previously showed that isoflurane GA induced the activation of NLRP3, the cleavage of caspase- 1 and the secretion of IL-1 $\beta$ and TNF- $\alpha$ in the hippocampus of aged mice (Wu et al., 2015a) and suggested that the NLRP3 inflammasome could be a promising molecular target for POCD treatment (Li et al., 2014; Wu et al., 2015a). MCC950 is a highly potent NLRP3 inhibitor and was first introduced as a specific anti-inflammatory compound in 2015 (Coll et al., 2015). The novel compound MCC950 has been clearly shown to be neuroprotective in multiple neurological disorders, including ischemic and degenerative diseases (Ye et al., 2017; Qi et al., 2018). However, the effects of MCC950 on GA-induced neuroinflammation and cognitive impairment are unknown.

Pyroptosis is a novel inflammatory form of programmed cell death. This type of cell death was recently characterized to be NLRP3 inflammasome-dependent and can be triggered in the canonical (caspase-1-mediated) and non-canonical (caspase-11-mediated) inflammasome signaling pathways (He et al., 2016a; Dempsey et al., 2017; Jiang et al., 2018). Accumulating evidence suggests that pyroptosis is involved in the pathogenesis of many infectious and non-infectious diseases (Shi et al., 2015; Aglietti and Dueber, 2017), and MCC950 can inhibit the NLRP3 inflammasome to further prevent pyroptosis (Mangan et al., 2018; Shen et al., 2018). However, little is known about the function of pyroptosis in GA-mediated neurotoxicity and cognitive deficits. Uncovering the mechanisms of this process may provide a novel avenue for POCD treatment. Thus, an effective intervention, MCC950, is emerging.

In the present study, we hypothesize that NLRP3 inflammasome-dependent pyroptosis is involved in the mechanism of isoflurane-induced cognitive impairment in aged mice and that inhibiting the NLRP3 inflammasome with MCC950 may ameliorate isoflurane-induced pyroptosis and cognitive impairment.

\section{MATERIALS AND METHODS}

\section{Animals}

Seventy-two 15-month-old male C57BL/6 mice were purchased from Jiangsu University, Zhenjiang, China. All experimental procedures and protocols were reviewed and approved by the Animal Investigation Ethics Committee of Jiangsu University and were done in accordance with the Guidelines for the Care and Use of Laboratory Animals from the National Institutes of Health, United States. The mice were housed in a room maintained under stable environmental conditions with a temperature of $22-24^{\circ} \mathrm{C}$ and a 12-h light/dark cycle.

\section{Experimental Protocols}

The mice were randomly assigned to one of the following four groups ( $n=18$ each): control, control + MCC950, isoflurane, and isoflurane + MCC950. MCC950 (10 mg/kg, China Peptides Co, Ltd, Shanghai, China) or phosphate-buffered saline (PBS, vehicle) was intraperitoneally administered to the mice in a volume of $0.4 \mathrm{~mL} / \mathrm{kg} 30 \mathrm{~min}$ before gas inhalation. The dose of MCC950 was chosen based on previous optimization showing that the intraperitoneal administration of MCC950 at $10 \mathrm{mg} / \mathrm{kg}$ presented neuroprotective effects in mice models (Ren et al., 2018; Xu et al., 2018; Zhai et al., 2018). Anesthesia was induced by placing the mice in an anesthetizing chamber prefilled with $1.8 \%$ isoflurane plus $30 \% \mathrm{O}_{2}$ for 10 min that was then changed to $1.5 \%$ isoflurane for $110 \mathrm{~min}$. For control experiments, 30\% $\mathrm{O}_{2}$ was delivered for $2 \mathrm{~h}$ at an identical flow rate ( $\mathrm{Wu}$ et al., 2015a; Wu et al., 2016). The composition of the chamber gas was continuously monitored using a Datex ${ }^{\mathrm{TM}}$ infrared analyzer (Capnomac, Helsinki, Finland). The mice were kept normothermic throughout the experiment.

Six mice in each group were decapitated $24 \mathrm{~h}$ after gas inhalation, and the brain was rapidly removed and separated into two halves for histochemical analysis and biochemical assays. The remaining 12 mice in each group were subjected to behavioral tests $24 \mathrm{~h}$ and 1 week after gas inhalation.

\section{Hematoxylin and Eosin (HE) Staining}

Half of each mouse brain ( $n=6$ for each group) underwent HE staining and immunohistochemical analysis $24 \mathrm{~h}$ after gas inhalation. Brain tissues were immersed in $4 \%$ paraformaldehyde and embedded using paraffin. The tissues were sliced into $4 \mathrm{~mm}$ sections until use.

HE staining was performed as follows: hematoxylin staining for $5 \mathrm{~min}, 75 \%$ hydrochloric acid alcohol solution for $30 \mathrm{~s}$ decoloring, eosin staining for $5 \mathrm{~min}$, and $90 \%$ ethanol for $35 \mathrm{~s}$ decoloring. Normal neurons have a relatively large cell body that is rich in cytoplasm with one or two large round nuclei, while damaged cells show shrunken cell bodies, pyknotic nuclei, dark cytoplasm, and many empty vesicles. Hippocampal neuronal damage was evaluated using a standard semi-quantitative scale (Tang et al., 2016). Briefly, grade $0=$ no damage to any hippocampal subregion, grade 1 = scattered neurons are damaged in the CA1 (cornu ammonis) subregion, grade $2=$ moderate numbers of neurons are damaged in the CA1 subregion $(<50 \%$ neurons damaged), grade $3=$ severe damage $(>50 \%$ of cells affected) to pyramidal cells in the CA1 subregion, and grade 4 = extensive cell damage in all hippocampal regions. Four random high-power (at $\times 400$ magnification) visual fields from each brain slice were checked. Evaluation of cells with nuclear pyknosis and morphologic abnormality was performed by two pathologists blind to the treatment groups.

\section{Immunohistochemistry}

Paraffin sections were then deparaffinized and hydrated using the following steps: $10 \mathrm{~min}$ in xylene twice; $5,10,10$, and $10 \mathrm{~min}$ in 100, 95, 85, and 70\% ethanol, respectively; and $5 \mathrm{~min}$ in PBS at room temperature repeated three times. Antigen retrieval was 
achieved by boiling the sections in $10 \mathrm{mM}$ sodium citrate for $10 \mathrm{~min}$ in a microwave oven. The sections were then washed with PBS three times and treated with $3 \% \mathrm{H}_{2} \mathrm{O}_{2}$-methanol for 15 min. Immunostaining was performed by incubation with antibody against NLRP3 (1:100; Servicebio Technology Co., Ltd., Wuhan, China) for $2 \mathrm{~h}$. The sections were then washed three times and incubated with secondary antibody labeled with horseradish peroxidase for $30 \mathrm{~min}$ at room temperature. Cells with brownish-yellow cytoplasm were counted as positive cells. The numbers of NLRP3 immunoreactive cells in the hippocampal CA1 region were counted and analyzed in four microscopic fields (at $\times 400$ magnification) by an investigator blinded to the treatment conditions.

\section{TUNEL Fluorescent Assay}

TUNEL assay was conducted with an in situ Cell Death Detection Kit (Roche Inc., Indianapolis, IN, United States) following the protocols. Sections were counterstained by Anti-NeuN Antibody (1:200, Merck Millipore, Hong Kong, China) for $3 \mathrm{~min}$. The sections were then washed with PBS three times and covered by microscopic glass with Antifade Mounting Medium (Beyotime Institute of Biotechnology, Shanghai, China) for further study. The numbers of TUNEL-positive cells in the hippocampal CA1 region were counted and analyzed (at $\times 400$ magnification) by an investigator blinded to the treatment conditions.

\section{Western Blotting Analysis}

The hippocampus from the other half of the brain $(n=6$ for each group) was isolated and homogenized for biochemical assays. Equal amounts of protein ( $35 \mu \mathrm{g} / \mathrm{lane})$ were electrophoretically separated and blotted onto nitrocellulose membranes. Protein levels were determined via incubation against antibody of NLRP3 (1:600; Servicebio Technology Co., Ltd.), ASC (1:200; Santa Cruz, CA, United States), caspase-1 (1:500; Servicebio Technology Co., Ltd.), gasdermin-D (GSDMD, 1:500; Abcam, United Kingdom), IL-1 $\beta$ (1:200; Santa Cruz, United States), IL18 (1:200; Santa Cruz, CA, United States), or $\beta$-actin $(1: 1,000$, Cell Signaling Technology, United States). Bands were visualized by enhanced chemiluminescence and quantified with the Image Quant Software (Syngene).

\section{Enzyme-Linked Immunosorbent Assay}

The quantifications of IL- $1 \beta$ and IL-18 in the hippocampus were performed using an enzyme-linked immunosorbent assay (ELISA) kit following the manufacturer's instructions (Servicebio Technology Co., Ltd).

\section{Open Field Test}

The open field test and fear conditioning test were conducted $24 \mathrm{~h}$ after gas inhalation in a sound-isolated room by a single investigator who was blinded to the group assignment. To evaluate the anxiety behavior and general locomotor activity, each mouse ( $n=12$ for each group) was gently placed in the center of a white plastic chamber $(40 \mathrm{~cm} \times 40 \mathrm{~cm} \times 40 \mathrm{~cm})$ for $5 \mathrm{~min}$, and exploratory behavior was automatically recorded by a video tracking system (XR-XZ301, Shanghai Softmaze

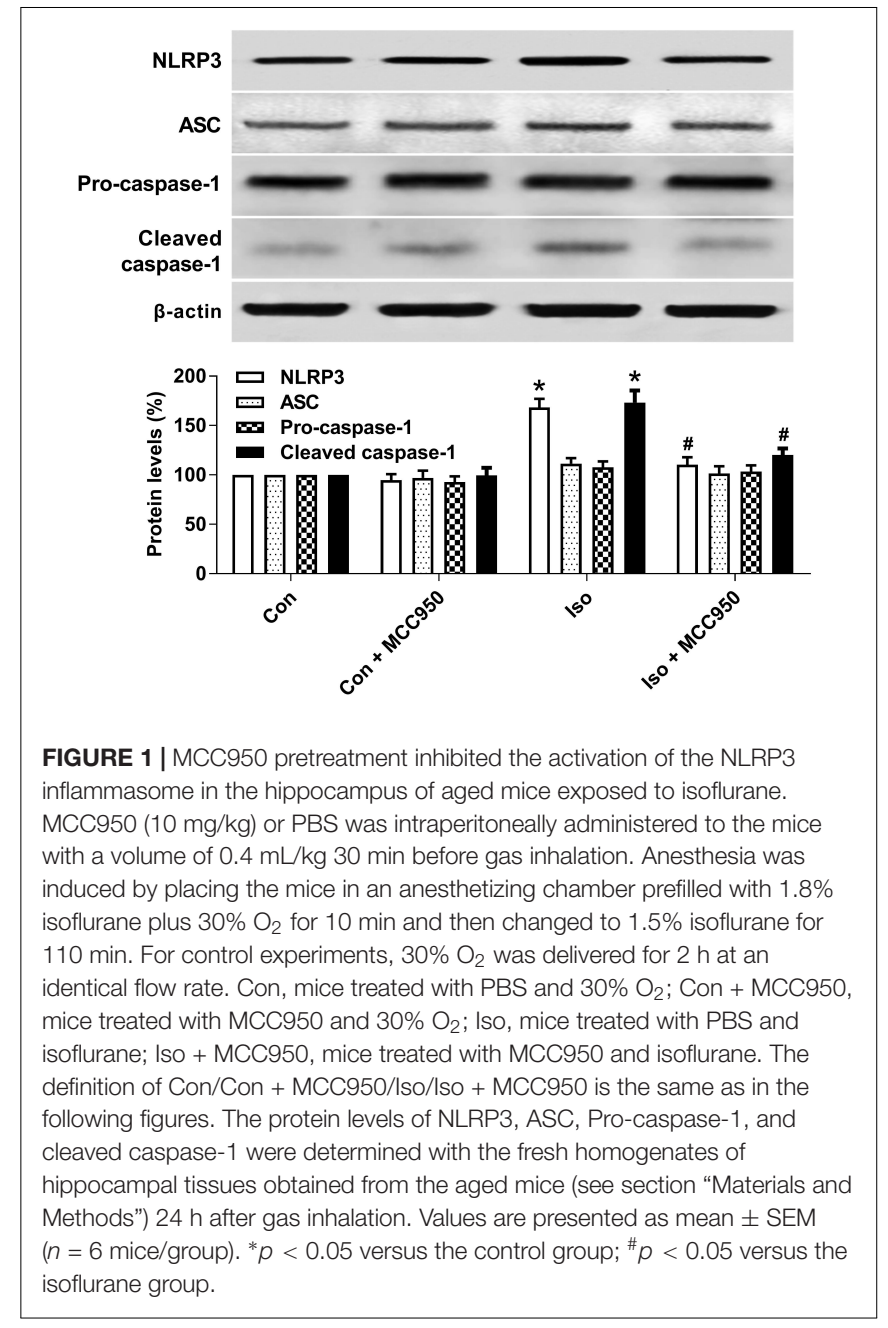

Information Technology Co., Ltd., Shanghai, China). After each test, the arena was cleaned with $75 \%$ alcohol to eliminate olfactory cues.

\section{Fear Conditioning Test}

To measure learning and memory abilities (Wu et al., 2015b), we employed the fear conditioning paradigm $(30 \mathrm{~cm}$ long $\times 26 \mathrm{~cm}$ wide $\times 22 \mathrm{~cm}$ high, XR-XC404, Shanghai Softmaze Information Technology Co., Ltd.). Each mouse was exposed to the conditioning chamber for 3 min of accommodation; one tone/foot shock pairing (tone, $30 \mathrm{~s}, 65 \mathrm{~dB}, 3 \mathrm{kHz}$; foot-shock, $3 \mathrm{~s}$, $0.75 \mathrm{~mA}$ ) was then delivered. The contextual fear conditioning test was performed $24 \mathrm{~h}$ later by placing each mouse back in the same test chamber for $5 \mathrm{~min}$ without any stimulation. Two hours later, each mouse was placed in a novel chamber that differed in shape, color, and smell, and the same tone was presented for $3 \mathrm{~min}$ without the foot shock to evaluate tone fear conditioning. Cognitive deficits in the test were assessed by measuring the length of "freezing behavior," which is defined as a completely immobile posture except for respiratory efforts. Freezing behaviors were automatically recorded by the video tracking system. 

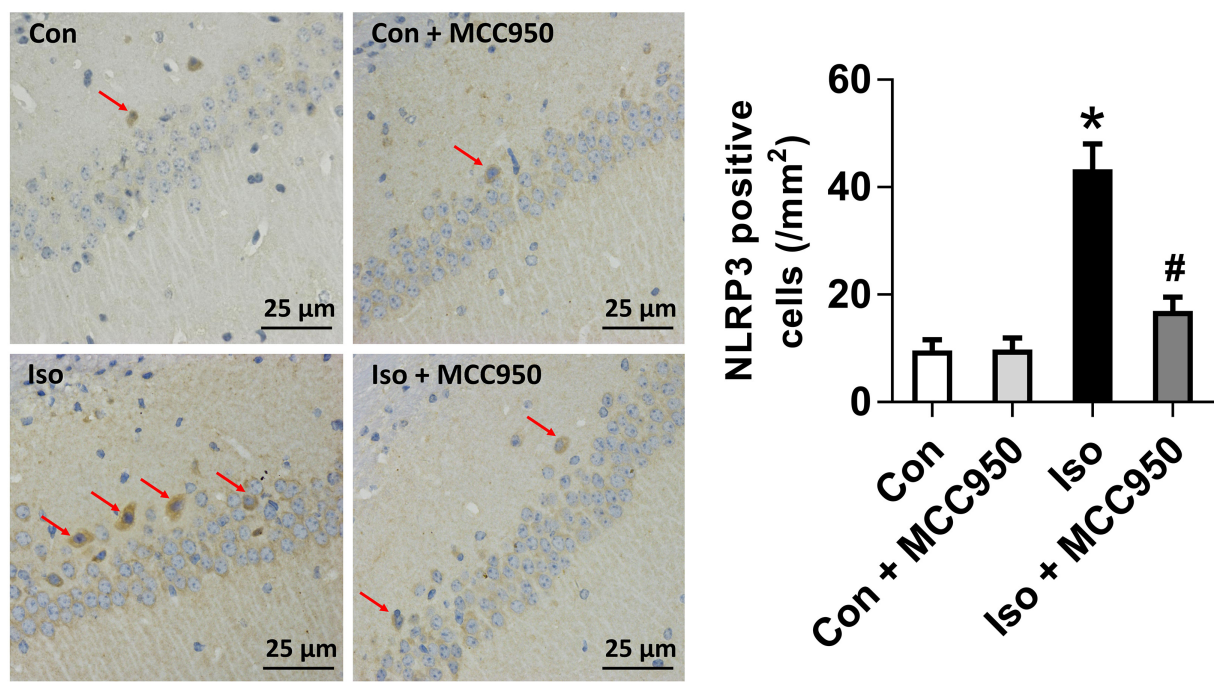

FIGURE 2 | The number of NLRP3-positive cells was diminished by MCC950 pretreatment in the hippocampal CA1 regions. Representative images of NLRP3 immunohistochemical $(\mathrm{HC})$ staining in the hippocampal CA1 region are shown. Cells with brownish-yellow cytoplasm are positive for NLRP3. Scale bar $=25 \mu \mathrm{m}$. Right panel presents statistical data from the four experimental groups. Values are presented as mean \pm SEM ( $n=6$ mice/group). ${ }^{*} p<0.05$ versus the control group; ${ }^{\#} p<0.05$ versus the isoflurane group.

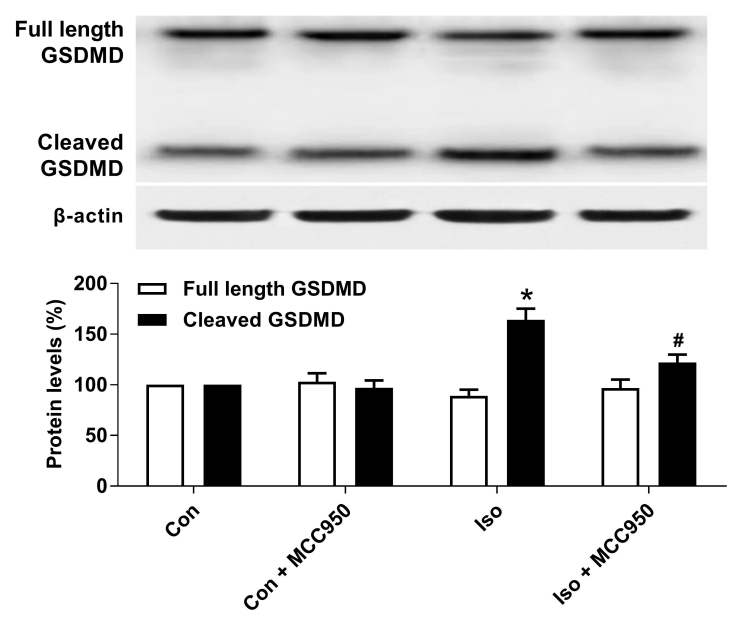

FIGURE 3 | Effects of isoflurane and MCC950 pretreatment on pyroptosis in the hippocampus of aged mice. Representative Western blotting and quantitative analysis of protein levels of full length GSDMD and cleaved GSDMD in the hippocampal tissues. Values are presented as mean \pm SEM ( $n=6$ mice/group). * $p<0.05$ versus the control group; ${ }^{\#} p<0.05$ versus the isoflurane group.

\section{Morris Water Maze (MWM) Tests}

One week after gas inhalation, the mice $(n=12$ for each group) were subjected to MWM (XR-XM101; Shanghai Softmaze Information Technology Co., Ltd.) to test their spatial learning and memory as previously described (Wu et al., 2017). Briefly, this consisted of two phases, a training phase for five consecutive days and a probe trial phase on day 6 . In the training phase, each mouse was allowed to face the pool wall in four random places $(\mathrm{N}, \mathrm{S}, \mathrm{E}$, and $\mathrm{W})$ in the pool to find the fixed platform.
Release positions were randomly predetermined. The trial was terminated once the mouse reached the platform. If the mouse failed to reach the platform within $60 \mathrm{~s}$, it would be guided to the platform and allowed to stay for $15 \mathrm{~s}$, and the latency was then recorded for $60 \mathrm{~s}$. In the probe test, a single-probe trial was conducted with the original platform removed $24 \mathrm{~h}$ after the last training session. Each mouse was allowed to swim for $60 \mathrm{~s}$ to test their memory (platform-crossing times and target quadrant traveling time).

\section{Statistical Analysis}

Data are presented as the mean \pm SEM and were analyzed by the Statistical Product for Social Sciences (SPSS; version 18.0, IL, United States). The difference between the groups was determined by one-way analysis of variance followed by the Bonferroni test. Comparisons for the spatial training sessions of MWM were performed by repeated two-way ANOVA followed by the LSD test. A $p$-value $<0.05$ was considered statistically significant.

\section{RESULTS}

\section{MCC950 Inhibits the Activation of the NLRP3 Inflammasome in the Hippocampus of Aged Mice Exposed to Isoflurane}

The NLRP3 inflammasome, a component of the inflammatory process, is highly expressed in various inflammatory diseases (Wu et al., 2015b; Mangan et al., 2018). MCC950 is the most specific and well characterized NLRP3 inhibitor to date and has been tested in a diverse array of NLRP3-involved inflammatory 


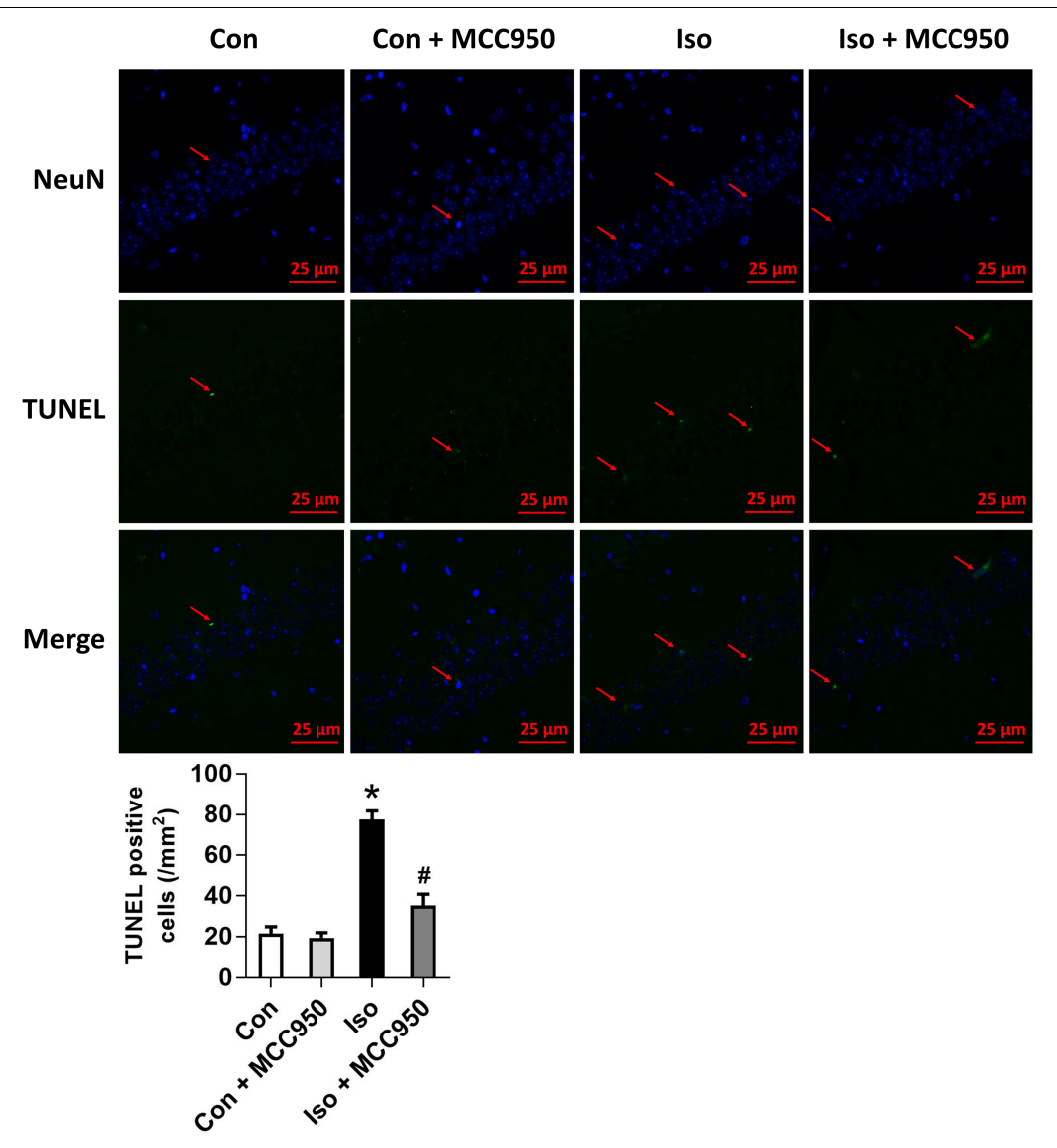

FIGURE 4 | Effects of isoflurane and MCC950 pretreatment on the number of TUNEL-positive cells in the hippocampal CA1 region. Representative images of TUNEL staining in the hippocampal CA1 region are shown. Green color indicates TUNEL-positive cells and blue indicates NeuN-stained nucleus. Scale bar $=25 \mu \mathrm{m}$. The lower panel shows statistical numbers of TUNEL-positive cells from the four experimental groups. Values are presented as mean \pm SEM ( $n=6$ mice/group). ${ }^{*} p<0.05$ versus the control group; ${ }^{*} p<0.05$ versus the isoflurane group.

diseases (Mangan et al., 2018; Shen et al., 2018). We previously showed that isoflurane induced NLRP3 inflammasome activation in the hippocampus of aged mice (Wu et al., 2015a). Here, we examined whether the inflammasome activation is inhibited by MCC950 treatment. Our results showed that the protein levels of NLRP3 and cleaved caspase-1 (Figure 1) and the number of NLRP3-positive cells (Figure 2) were increased $24 \mathrm{~h}$ after $2 \mathrm{~h}$ isoflurane exposure. Notably, the increases were reversed by the administration of MCC950 (Figures 1, 2). The adaptor protein ASC and pro-caspase-1 showed no differences among the four groups (Figure 1). Our results indicate that isoflurane-induced NLRP3 inflammasome activation is successfully inhibited by MCC950 administration.

\section{Inhibiting the NLRP3 Inflammasome With MCC950 Ameliorates Pyroptosis in the Hippocampus of Aged Mice Exposed to Isoflurane}

Pyroptosis was recently characterized to be NLRP3 inflammasome-dependent. NLRP3 inflammasome activation governs the cleavage of caspase-1, resulting in the maturation of effector pro-inflammatory cytokines and mediating pyroptosis (He et al., 2016a; Dempsey et al., 2017; Jiang et al., 2018). Recent progress has identified the protein GSDMD as the substrate of active caspase-1 and caspase-11 and the executor of proptosis (Kayagaki et al., 2015; Shi et al., 2015). Active caspase-1 (canonical inflammasome pathway) or caspase-11 (non-canonical inflammasome pathway) enzymatically cleaves GSDMD into two fragments (the $\mathrm{N}$ domain and C domain) (Shi et al., 2015, 2017; Gaidt and Hornung, 2016; Sborgi et al., 2016; Aglietti and Dueber, 2017). Furthermore, the GSDMD-N domain forms pores on lipid membranes and induces pyroptosis through cell membrane disruption. Here, we showed that isoflurane GA induced an increase in cleaved GSDMD but not full length GSDMD in the hippocampus of aged mice (Figure 3), implying that pyroptosis was activated during isoflurane GA. In addition, the TUNEL assay showed that the percentage of dead neurons in the isoflurane group was higher than that in the control group (Figure 4). Interestingly, MCC950 pretreatment attenuated the pyroptotic cell death induced by isoflurane GA (Figures 3, 4). These results suggest that inhibiting the NLRP3 inflammasome with MCC950 ameliorates pyroptosis in the hippocampus of aged mice exposed to isoflurane. 
A

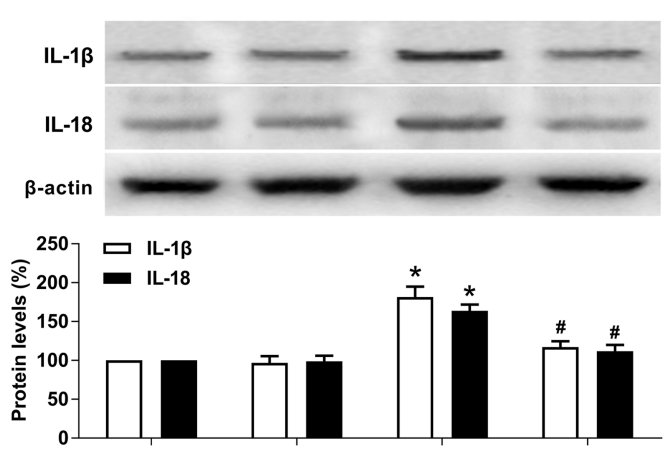

B

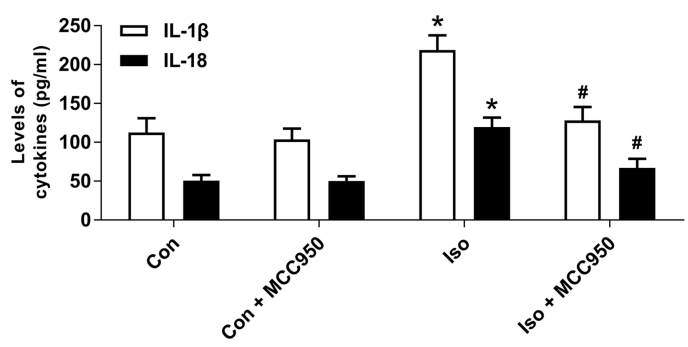

FIGURE 5 | MCC950 pretreatment suppressed the release of inflammatory cytokines in the hippocampus of aged mice exposed to isoflurane.

(A) Western blotting analyses of IL-1 $\beta$ and IL-18 levels. (B) ELISA assays of $\mathrm{IL}-1 \beta$ and IL-18 levels. Values are presented as mean \pm SEM $(n=6$ mice/group). ${ }^{*} p<0.05$ versus the control group; ${ }^{\#} p<0.05$ versus the isoflurane group.

\section{MCC950 Suppresses the Release of Inflammatory Cytokines in the Hippocampus of Aged Mice Exposed to Isoflurane}

Pyroptotic cell death involves the formation of a plasma membrane pore, cell swelling, and rupture of the plasma membrane, which results in the release of abundant inflammatory factors (Shi et al., 2015, 2017; Aglietti and Dueber, 2017). Our results showed that the inflammatory cytokines IL- $1 \beta$ and IL18 (Figure 5) were increased $24 \mathrm{~h}$ after isoflurane exposure in the hippocampus of the aged mice, while MCC950 pretreatment significantly suppressed the secretion of IL-1 $\beta$ and IL-18 (Figure 5). Therefore, our results suggest that inhibiting NLRP3 inflammasome-dependent pyroptosis with MCC950 suppresses the secretion of inflammatory cytokines in the hippocampus of aged mice exposed to isoflurane.

\section{MCC950 Reverses Neuronal Morphological Damage in the Hippocampus of Aged Mice Exposed to Isoflurane}

To evaluate the neuroprotective effects of MCC950 on isofluraneinduced neuronal morphological damage, we conducted HE staining on brain sections of the hippocampal CA1 region $24 \mathrm{~h}$ after gas inhalation. The mice in the isoflurane group showed the most condensed cell bodies, as well as most extensive karyopyknosis and karyolysis (Figure 6). In contrast, the mice with MCC950 pretreatment showed significant decreases in abnormal neurons and neuronal damage scores (Figure 6). This result suggests that MCC950 protects hippocampal neurons from isoflurane-induced morphological damage.

\section{MCC950 Attenuates Isoflurane-Induced Cognitive Impairment in Aged Mice}

To further evaluate the neuroprotective effects of MCC950 on isoflurane-induced cognitive impairment, the open field, fear conditioning and MWM tests were performed. The open field test showed no difference among the four groups in locomotor activity as reflected by the total distance (Figure 7A) and time spent in the center (Figure 7B), which excluded the possibility that locomotor activity per se affected the results in the fear conditioning and MWM tests.

The fear conditioning test showed a decreased percentage of freezing time in the $24 \mathrm{~h}$ context test, which reflected impairment of hippocampus-dependent memory in isofluranetreated mice compared to that in the control mice (Figure 7C). The MWM tests showed a long escape latency in the training test (Figure 7E) as well as decreased target quadrant time (Figure 7F) and crossing-platform times (Figure 7G) in the probe trial in the isoflurane-treated mice. Interestingly, all of the changes in the behavioral tests (Figure 7) were counteracted by MCC950 pretreatment. These results indicate that isoflurane GA for $2 \mathrm{~h}$ led to cognitive impairment in the aged mice and that MCC950 $(10 \mathrm{mg} / \mathrm{kg})$ intraperitoneal treatment has a therapeutic effect on isoflurane-induced cognitive impairment.

\section{DISCUSSION}

We previously demonstrated that isoflurane GA induced hippocampal NLRP3 inflammasome activation and cognitive deficits in aged mice ( $\mathrm{Wu}$ et al., 2015a). In the present study, we showed that the isoflurane-induced activation of the NLRP3 inflammasome triggered neuronal damage in a pyroptotic manner. Pretreatment with NLRP3 inflammasome inhibitor MCC950 significantly suppressed the expressions of NLRP3 inflammasome and cleaved caspase- 1 and the associated release of the inflammatory cytokines IL-1 $\beta$ and IL-18 in the hippocampus of aged mice. Moreover, MCC950 protected aged mice from isoflurane-induced neuronal damage and cognitive impairment. Our results collectively suggest that NLRP3 inflammasome-dependent pyroptosis plays a negative role in the pathogenesis of isoflurane-induced cognitive impairment in aged mice and that MCC950 acts as a potential therapeutic compound for the treatment of POCD.

The NLRP3 inflammasome is a critical component of inflammasomes that functions through an interaction with apoptosis-associated speck-like protein (ASC) and the subsequent recruitment of the precursor form of caspase-1, leading to the cleavage of caspase- 1 and the maturation and secretion of IL-1 $\beta$ and IL-18 (He et al., 2016a; 

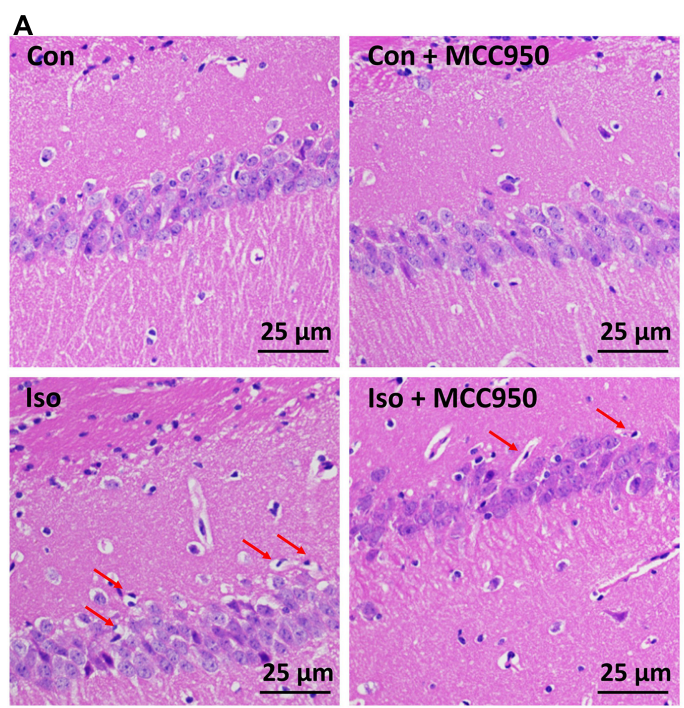

B

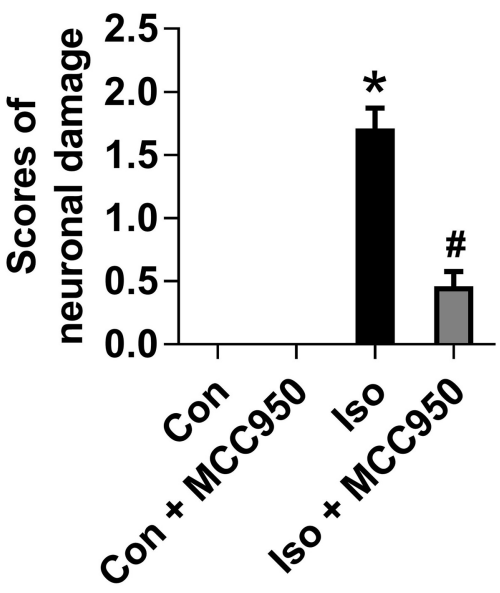

FIGURE 6 | MCC950 pretreatment reversed isoflurane-induced neuronal morphological damage in the hippocampus of aged mice. (A) Representative pictures of HE staining in the hippocampal CA1 region are shown. Red arrows: Cells arranged in a disorderly manner, cytoplasm and nucleolus deeply dyed with nuclear pyknosis. Scale bar $=25 \mu \mathrm{m}$. (B) The right panel shows statistical scores of neuronal damage. Values are presented as mean \pm SEM $\left(n=6\right.$ mice/group). ${ }^{*} p<0.05$ versus the control group; ${ }^{*} p<0.05$ versus the isoflurane group.

A

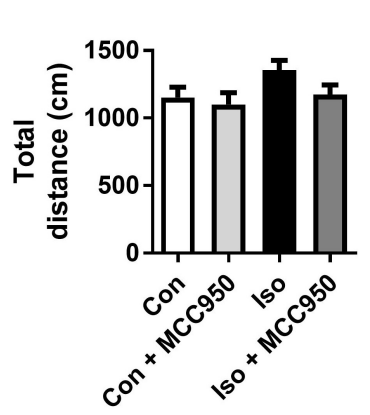

E

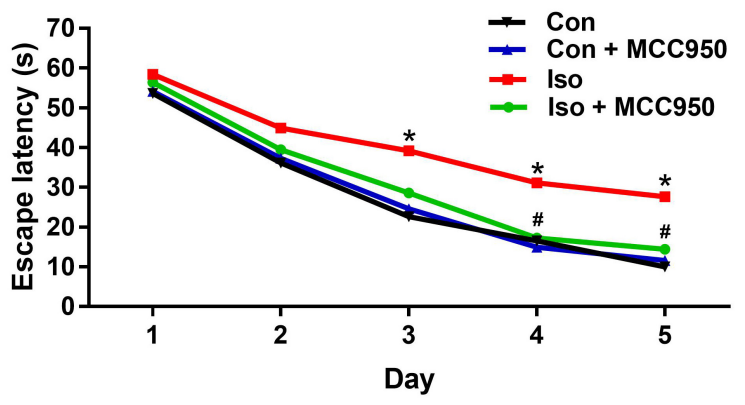

B

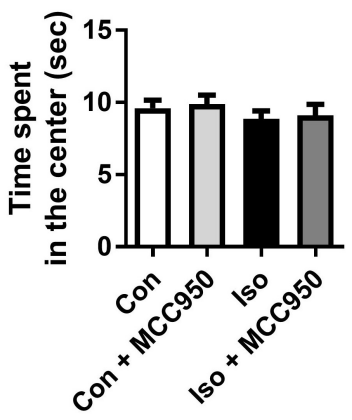

$\mathbf{F}$
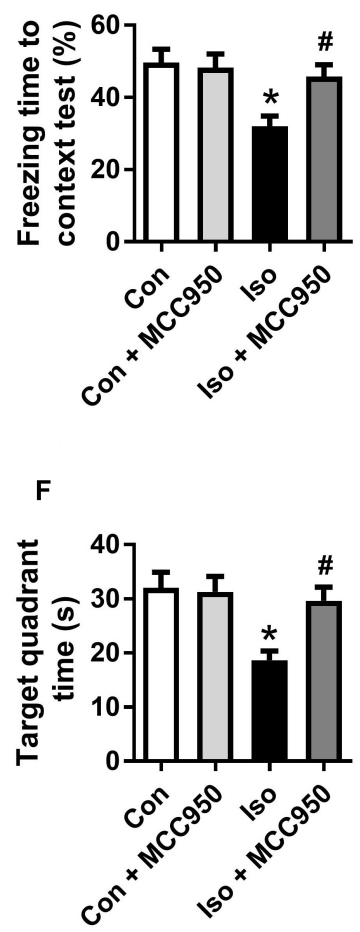

D

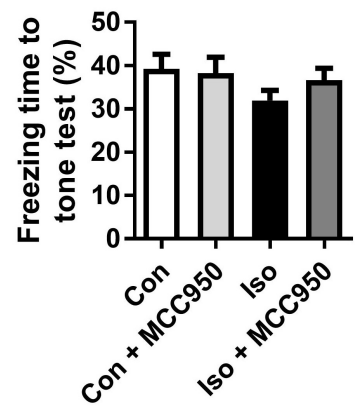

G

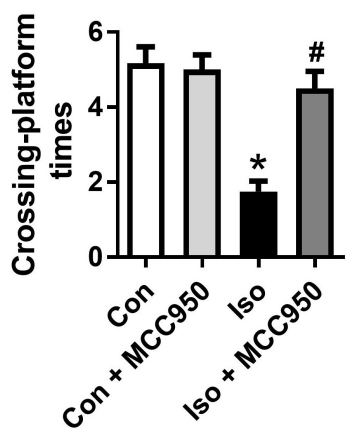

FIGURE 7 | MCC950 pretreatment prevented isoflurane-induced cognitive impairment in aged mice. (A) Total distance traveled and (B) time spent in the center in the open field test. (C) Freezing time to context and (D) freezing time to tone in the fear conditioning test. (E) Escape latency for every day during the spatial training of MWM. (F) Time spent in the target quadrant and (G) crossing-platform times in the probe trial of MWM. Values are presented as mean \pm SEM $(n=12$ mice/group). ${ }^{*} p<0.05$ versus the control group; ${ }^{\#} p<0.05$ versus the isoflurane group. The procedures of the behavioral tests are in the section "Materials and Methods." MWM, Morris water maze. 
Dempsey et al., 2017; Jiang et al., 2018). Consistent with previous studies, we found that isoflurane induced the upregulation of NLRP3, the cleavage of caspase-1, and the secretion of IL- $1 \beta$ and IL-18 in the hippocampus of aged mice. Unexpectedly, isoflurane did not increase the expression of ASC in the present study. It is possible that other proteins are involved in the formation of the NLRP3 inflammasome, such as NIMA-related kinases (He et al., 2016b) and protein kinase D (Zhang et al., 2017). Moreover, activated NLRP3 inflammasome-caspase-1 can cleave GSDMD into the GSDMD-N domain, which further forms pores on lipid membranes and induces pyroptosis (Shi et al., 2015, 2017; Gaidt and Hornung, 2016; Sborgi et al., 2016; Aglietti and Dueber, 2017). Thus, our study also showed that cleaved GSDMD, but not full length GSDMD, was upregulated in the hippocampus of aged mice exposed to isoflurane. Our data provide preliminary evidence that pyroptosis is likely involved in GA-induced neuroinflammation.

Activation of the NLRP3 inflammasome and pyroptosis may cause neuronal damage, cognitive dysfunction, and even death (Bordon, 2012; Tan et al., 2014; Ding et al., 2018). The hippocampus is known to be critical for contextual and spatial memory, while acute pathological conditions, such as ischemia, hypoglycemia and other neurological conditions, can cause damage to the hippocampus (Squire, 1992; Bartsch and Wulff, 2015; Wu et al., 2015b). This acute damage results in the selective cell death of hippocampal CA1 neurons, whereas the dentate gyrus (DG), CA3, and most cortical neurons appear to be more resistant (Bartsch and Wulff, 2015; Bartsch et al., 2015). Thus, we examined neuronal damage in the hippocampal CA1 region in the present study and found that mice in the isoflurane group exhibited massively injured neurons in this region. Moreover, the mice in the isoflurane group performed significantly worse in the contextual fear conditioning test and MWM tests than those in the control group, showing that hippocampus-dependent contextual memory and spatial learning and memory were impaired. Altogether, previous studies as well as our study suggest that NLRP3 inflammasome-dependent pyroptosis is a key regulator of the underlying mechanisms of isoflurane-induced neuronal damage and cognitive impairment in aged mice.

MCC950 is a newly developed, selective, small-molecule NLRP3 inflammasome inhibitor that can freely cross the bloodbrain barrier (Chen et al., 2017; Qi et al., 2018). Zhai et al. (2018) recently reported that the intraperitoneal administration

\section{REFERENCES}

Aglietti, R. A., and Dueber, E. C. (2017). Recent insights into the molecular mechanisms underlying pyroptosis and gasdermin family functions. Trends Immunol. 38, 261-271. doi: 10.1016/j.it.2017.01.003

Bartsch, T., Dohring, J., Reuter, S., Finke, C., Rohr, A., Brauer, H., et al. (2015). Selective neuronal vulnerability of human hippocampal CA1 neurons: lesion evolution, temporal course, and pattern of hippocampal damage in diffusionweighted MR imaging. J. Cereb. Blood Flow Metab. 35, 1836-1845. doi: 10.1038/ jcbfm.2015.137

Bartsch, T., and Wulff, P. (2015). The hippocampus in aging and disease: from plasticity to vulnerability. Neuroscience 309, 1-16. doi: 10.1016/j.neuroscience. 2015.07.084 of MCC950 (10 mg/kg) could downregulate the hippocampal NLRP3, ASC, and IL-1 $\beta$ expression levels and ameliorate cognitive impairment in diabetic $\mathrm{db} / \mathrm{db}$ mice. In line with these data, our study showed that MCC950 (10 mg/kg) intraperitoneal treatment was efficacious in ameliorating cognitive impairment in aged mice exposed to isoflurane. In addition, histochemical and biochemical findings supported that MCC950 protected aged mice against hippocampal NLRP3 inflammasome activation, inflammatory cytokines secretion, pyroptotic cell death, and neuronal morphological damage. These findings provided evidence that the administration of the specific NLRP3 inhibitor MCC950 ameliorates the pyroptosis and cognitive impairment caused by isoflurane anesthesia in the aged mice.

\section{CONCLUSION}

This is the first study to our knowledge to show that pyroptosis is involved in isoflurane-induced cognitive impairment in aged mice and that inhibiting the NLRP3 inflammasome with MCC950 ameliorates the neuroinflammation, pyroptosis, neuronal damage, and cognitive impairment caused by isoflurane anesthesia in these mice. This work suggests a new strategy for reversing age- and/or neuroinflammation-related impairment in GA-induced neurotoxicity and cognitive deficits, supporting the notion that MCC950 may have therapeutic benefits for elderly patients with POCD.

\section{AUTHOR CONTRIBUTIONS}

YF and LD performed the experiments and acquired the data. YF, LD, QF, ZZ, and JZ analyzed and interpreted the data. JW conceived and designed the experiments. YF and JW wrote the manuscript. YF, LD, QF, ZZ, JZ, and GL contributed to the acquisition and analysis of data. All authors read and approved the final manuscript.

\section{FUNDING}

This work was supported by the National Nature Science Foundation of China, Grant Nos. 81271216 and 81471105.

Berger, M., Nadler, J. W., Browndyke, J., Terrando, N., Ponnusamy, V., Cohen, H. J., et al. (2015). Postoperative cognitive dysfunction: minding the gaps in our knowledge of a common postoperative complication in the elderly. Anesthesiol. Clin. 33, 517-550. doi: 10.1016/j.anclin.2015.05.008

Bordon, Y. (2012). Mucosal immunology: inflammasomes induce sepsis following community breakdown. Nat. Rev. Immunol. 12, 400-401. doi: 10.1038/nri 3235

Chen, W., Foo, S. S., Zaid, A., Teng, T. S., Herrero, L. J., Wolf, S., et al. (2017). Specific inhibition of NLRP3 in chikungunya disease reveals a role for inflammasomes in alphavirus-induced inflammation. Nat. Microbiol. 2, 1435-1445. doi: 10.1038/s41564-017-0015-4

Coll, R. C., Robertson, A. A., Chae, J. J., Higgins, S. C., Munoz-Planillo, R., Inserra, M. C., et al. (2015). A small-molecule inhibitor of the NLRP3 inflammasome for 
the treatment of inflammatory diseases. Nat. Med. 21, 248-255. doi: 10.1038/ nm.3806

Dempsey, C., Rubio Araiz, A., Bryson, K. J., Finucane, O., Larkin, C., Mills, E. L., et al. (2017). Inhibiting the NLRP3 inflammasome with MCC950 promotes non-phlogistic clearance of amyloid-beta and cognitive function in APP/PS1 mice. Brain Behav. Immun. 61, 306-316. doi: 10.1016/j.bbi.2016.12.014

Ding, H. G., Deng, Y. Y., Yang, R. Q., Wang, Q. S., Jiang, W. Q., Han, Y. L., et al. (2018). Hypercapnia induces IL-1beta overproduction via activation of NLRP3 inflammasome: implication in cognitive impairment in hypoxemic adult rats. J. Neuroinflammation 15:4. doi: 10.1186/s12974-017-1051-y

Gaidt, M. M., and Hornung, V. (2016). Pore formation by GSDMD is the effector mechanism of pyroptosis. EMBO J. 35, 2167-2169. doi: 10.15252/embj. 201695415

He, Y., Hara, H., and Nunez, G. (2016a). Mechanism and regulation of NLRP3 inflammasome activation. Trends Biochem. Sci. 41, 1012-1021. doi: 10.1016/j. tibs.2016.09.002

He, Y., Zeng, M. Y., Yang, D., Motro, B., and Nunez, G. (2016b). NEK7 is an essential mediator of NLRP3 activation downstream of potassium efflux. Nature 530, 354-357. doi: 10.1038/nature16959

Hovens, I. B., Schoemaker, R. G., Van Der Zee, E. A., Absalom, A. R., Heineman, E., and Van Leeuwen, B. L. (2014). Postoperative cognitive dysfunction: involvement of neuroinflammation and neuronal functioning. Brain Behav. Immun. 38, 202-210. doi: 10.1016/j.bbi.2014.02.002

Jiang, D. L., Chen, S., Sun, R. Y., Zhang, X., and Wang, D. (2018). The NLRP3 inflammasome: role in metabolic disorders and regulation by metabolic pathways. Cancer Lett. 419, 8-19. doi: 10.1016/j.canlet.2018.01.034

Kayagaki, N., Stowe, I. B., Lee, B. L., O'rourke, K., Anderson, K., Warming, S., et al. (2015). Caspase-11 cleaves gasdermin D for non-canonical inflammasome signalling. Nature 526, 666-671. doi: 10.1038/nature15541

Li, X. M., Zhou, M. T., Wang, X. M., Ji, M. H., Zhou, Z. Q., and Yang, J. J. (2014). Resveratrol pretreatment attenuates the isoflurane-induced cognitive impairment through its anti-inflammation and -apoptosis actions in aged mice. J. Mol. Neurosci. 52, 286-293. doi: 10.1007/s12031-013-0141-2

Mangan, M. S. J., Olhava, E. J., Roush, W. R., Seidel, H. M., Glick, G. D., and Latz, E. (2018). Targeting the NLRP3 inflammasome in inflammatory diseases. Nat. Rev. Drug Discov. 17, 588-606. doi: 10.1038/nrd.2018.97

Moskowitz, E. E., Overbey, D. M., Jones, T. S., Jones, E. L., Arcomano, T. R., Moore, J. T., et al. (2017). Post-operative delirium is associated with increased 5-year mortality. Am. J. Surg. 214, 1036-1038. doi: 10.1016/j.amjsurg.2017.08.034

Qi, Y., Klyubin, I., Cuello, A. C., and Rowan, M. J. (2018). NLRP3-dependent synaptic plasticity deficit in an Alzheimer's disease amyloidosis model in vivo. Neurobiol. Dis. 114, 24-30. doi: 10.1016/j.nbd.2018.02.016

Ren, H., Kong, Y., Liu, Z., Zang, D., Yang, X., Wood, K., et al. (2018). Selective NLRP3 (Pyrin Domain-Containing Protein 3) inflammasome inhibitor reduces brain injury after intracerebral hemorrhage. Stroke 49, 184-192. doi: 10.1161/ STROKEAHA.117.018904

Sborgi, L., Ruhl, S., Mulvihill, E., Pipercevic, J., Heilig, R., Stahlberg, H., et al. (2016). GSDMD membrane pore formation constitutes the mechanism of pyroptotic cell death. EMBO J. 35, 1766-1778. doi: 10.15252/embj.201694696

Shen, H. H., Yang, Y. X., Meng, X., Luo, X. Y., Li, X. M., Shuai, Z. W., et al. (2018). NLRP3: a promising therapeutic target for autoimmune diseases. Autoimmun. Rev. 17, 694-702. doi: 10.1016/j.autrev.2018.01.020

Shi, J., Gao, W., and Shao, F. (2017). Pyroptosis: gasdermin-mediated programmed necrotic cell death. Trends Biochem. Sci. 42, 245-254. doi: 10.1016/j.tibs.2016. 10.004

Shi, J., Zhao, Y., Wang, K., Shi, X., Wang, Y., Huang, H., et al. (2015). Cleavage of GSDMD by inflammatory caspases determines pyroptotic cell death. Nature 526, 660-665. doi: 10.1038/nature15514
Skvarc, D. R., Berk, M., Byrne, L. K., Dean, O. M., Dodd, S., Lewis, M., et al. (2018). Post-operative cognitive dysfunction: an exploration of the inflammatory hypothesis and novel therapies. Neurosci. Biobehav. Rev. 84, 116-133. doi: 10. 1016/j.neubiorev.2017.11.011

Squire, L. R. (1992). Memory and the hippocampus: a synthesis from findings with rats, monkeys, and humans. Psychol. Rev. 99, 195-231. doi: 10.1037/0033-295X. 99.2.195

Tan, M. S., Tan, L., Jiang, T., Zhu, X. C., Wang, H. F., Jia, C. D., et al. (2014). Amyloid-beta induces NLRP1-dependent neuronal pyroptosis in models of Alzheimer's disease. Cell Death Dis. 5:e1382. doi: 10.1038/cddis.2014.348

Tang, Y., Liu, X., Zhao, J., Tan, X., Liu, B., Zhang, G., et al. (2016). Hypothermiainduced ischemic tolerance is associated with Drp1 inhibition in cerebral ischemia-reperfusion injury of mice. Brain Res. 1646, 73-83. doi: 10.1016/j. brainres.2016.05.042

Umholtz, M., and Nader, N. D. (2017). Anesthetic immunomodulation of the neuroinflammation in postoperative cognitive dysfunction. Immunol. Invest. 46, 805-815. doi: 10.1080/08820139.2017.1373898

Wu, J., Hao, S., Sun, X. R., Zhang, H., Li, H., Zhao, H., et al. (2017). Elamipretide (SS-31) ameliorates isoflurane-induced long-term impairments of mitochondrial morphogenesis and cognition in developing rats. Front. Cell Neurosci. 11:119. doi: 10.3389/fncel.2017.00119

Wu, J., Li, H., Sun, X., Zhang, H., Hao, S., Ji, M., et al. (2015a). A Mitochondriontargeted antioxidant ameliorates isoflurane-induced cognitive deficits in aging mice. PLoS One 10:e0138256. doi: 10.1371/journal.pone.0138256

Wu, J., Zhang, M., Hao, S., Jia, M., Ji, M., Qiu, L., et al. (2015b). Mitochondriatargeted peptide reverses mitochondrial dysfunction and cognitive deficits in sepsis-associated encephalopathy. Mol. Neurobiol. 52, 783-791. doi: 10.1007/ s12035-014-8918-Z

Wu, J., Zhang, M., Li, H., Sun, X., Hao, S., Ji, M., et al. (2016). BDNF pathway is involved in the protective effects of SS-31 on isoflurane-induced cognitive deficits in aging mice. Behav. Brain Res. 305, 115-121. doi: 10.1016/j.bbr.2016. 02.036

Xu, X., Yin, D., Ren, H., Gao, W., Li, F., Sun, D., et al. (2018). Selective NLRP3 inflammasome inhibitor reduces neuroinflammation and improves long-term neurological outcomes in a murine model of traumatic brain injury. Neurobiol. Dis. 117, 15-27. doi: 10.1016/j.nbd.2018.05.016

Ye, X., Shen, T., Hu, J., Zhang, L., Zhang, Y., Bao, L., et al. (2017). Purinergic 2X7 receptor/NLRP3 pathway triggers neuronal apoptosis after ischemic stroke in the mouse. Exp. Neurol. 292, 46-55. doi: 10.1016/j.expneurol.2017.03.002

Zhai, Y., Meng, X., Ye, T., Xie, W., Sun, G., and Sun, X. (2018). Inhibiting the NLRP3 inflammasome activation with mcc950 ameliorates diabetic encephalopathy in $\mathrm{db} / \mathrm{db}$ Mice. Molecules 23:E522. doi: 10.3390/ molecules23030522

Zhang, Z., Meszaros, G., He, W. T., Xu, Y., De Fatima Magliarelli, H., Mailly, L., et al. (2017). Protein kinase D at the Golgi controls NLRP3 inflammasome activation. J. Exp. Med. 214, 2671-2693. doi: 10.1084/jem.20162040

Conflict of Interest Statement: The authors declare that the research was conducted in the absence of any commercial or financial relationships that could be construed as a potential conflict of interest.

Copyright (c) $2018 \mathrm{Fan}, \mathrm{Du}, \mathrm{Fu}$, Zhou, Zhang, Li and Wu. This is an open-access article distributed under the terms of the Creative Commons Attribution License (CC BY). The use, distribution or reproduction in other forums is permitted, provided the original author(s) and the copyright owner(s) are credited and that the original publication in this journal is cited, in accordance with accepted academic practice. No use, distribution or reproduction is permitted which does not comply with these terms. 